\title{
Estilos de Citas y Referencias Bibliográficas en Revistas Odontológicas SciELO: Un Elemento Crítico de la Escritura Científica
}

\author{
Styles for Citations and Referencing in Dental SciELO Journals: \\ A Critical Element of Scientific Writing
}

Mario Cantín ${ }^{* * *} \&$ Priscila Chuhuaicura***

CANTíN, M. \& CHUHUAICURA, P. Estilos de citas y referencias bibliográficas en revistas odontológicas SciELO: Un elemento crítico de la escritura científica. Int. J. Odontostomat., 10(2):349-357, 2016.

RESUMEN: Uno de los problemas más comunes en las revistas científicas, es el incumplimiento de los estilos al hacer citas y escribir las referencias bibliográficas, debido al descuido o bien al desconocimiento de los diferentes sistemas existentes y sus características. El objetivo fue revisar los estilos de citas y referencias utilizados por revistas odontológicas indexadas en la red SciELO, con el fin describir los más utilizados y evidenciar las diferencias existentes entre las revistas. Fueron revisados 100 artículos pertenecientes a 20 revistas diferentes, seleccionados aleatoriamente por afijación proporcional. El estilo más utilizado para las citas y referencias bibliográficas correspondió al sistema Vancouver. Sólo una revista, International Journal of Odontostomatology publica sus artículos bajo el sistema Harvard. Estos estilos sirven como un sello de las revistas, pero constituyen una carga de trabajo adicional significativa, ya que la atención a los detalles que se requieren para asegurar que las citas y referencias se ajusten a los estilos individuales de las revistas es fundamental. Cualquiera sea el estilo de la revista, es importante utilizarlo de una manera consistente. La responsabilidad de la exactitud en las citas y referencias bibliográficas es de los autores, por lo que deben preocuparse de ello al redactar el artículo, en su revisión final previo al envío, así como en las pruebas de imprenta.

PALABRAS CLAVE: referencias bibliográficas, estilos de referencias, citas, Vancouver, Harvard, escritura científica.

\section{INTRODUCCIÓN}

Al escribir un artículo científico, hay muchas barreras que superar. Una de ellas, que requiere tiempo, esfuerzo y atención, es la correcta citación y referenciación de los artículos que darán el soporte y evidencia en cualquier investigación. Los diferentes sistemas o estilos exigidos por las revistas, tanto para citas en el texto como para las referencias bibliográficas, demandan una atención significativa hasta el último detalle, lo cual constituye un desafío para la publicación (Harries et al., 2013).

Las citas y referencias bibliográficas tienen un rol importante en cualquier trabajo científico; proporcionan una manera rápida de revisar antecedentes his- tóricos, documentan investigaciones que apoyan hechos $u$ opiniones expresadas en el artículo, reconocen el trabajo de otros y le entregan al lector fuentes de información adicional sobre el tema. Así, estas no sólo deben ser pertinentes, si no que también exactas y coherentes (Laskin, 1996; Masic, 2013), donde todos los autores de algún artículo son responsables de la exactitud e integridad de las referencias (Riordan, 2012). Las citas permiten identificar brevemente en el texto la fuente de información, mientras que las referencias bibliográficas proporcionan la información completa y exacta para cada fuente, siendo mutuamente necesarias para su existencia en un manuscrito, lo citado debe estar referenciado y cada referencia debe

\footnotetext{
* Centro de Investigación CIMA, Facultad de Odontología, Universidad de La Frontera, Temuco, Chile.

* Centro de Investigación en Ciencias Biomédicas, Universidad Autónoma de Chile, Temuco, Chile.

${ }^{* * *}$ Estudiante. Escuela de Odontología, Facultad de Odontología, Universidad de La Frontera, Chile.

Financiado (parcialmente) por la Dirección de Investigación, Universidad de La Frontera, Proyecto DI16-2021.
} 
ser citada. Casi sin importar el estilo, la ubicación de las referencias bibliográficas al final del artículo es una de las pocas formalidades que se respetan universalmente, basada en un consenso lógico (Reyes, 2001).

Existen varios sistemas para la citación y referenciación. Dentro de los más comunes, están los sistemas "autor-fecha" (tal como el sistema de Harvard, APA, etc.) y sistemas "numéricos" (tales como Vancouver, CSA, IEEE, etc.). Aunque estos estilos difieren en su representación, ellos proporcionan la misma información y sirven para el mismo propósito (Kali, 2016). El sistema de una revista científica depende de la disciplina en la que escribe el autor (Masic), y cada revista exigirá uniformidad de formato (y sistema) a los artículos que reciben, los cuales comúnmente se especifican en sus "instrucciones a los autores", declarando cuál es el sistema que aceptan para las citas y referencias bibliográficas, según el grupo normativo al que estén adscritas.

Uno de los problemas más comunes en las revistas científicas, es el incumplimiento de formatos al hacer citas y escribir las referencias bibliográficas de acuerdo a las instrucciones de la revista de destino (Peh \& Ng), esto debido al descuido o bien al desconocimiento de los diferentes sistemas y estilos que poseen las diferentes revistas. Además, cuando se encuentran imprecisiones en las citas y referencias bibligráficas, se levanta sospechas sobre la exactitud y calidad del resto del artículo (Laskin). En la literatura odontológica, es infrecuente leer guías o temas relacionados a este fundamental aspecto de la escritura, comunicación e investigación científica.

El objetivo fue revisar los diferentes estilos de citas y referencias utilizados por las revistas odontológicas indexadas en la base SciELO, con el fin describir los más comunes y evidenciar las diferencias existentes entre las revistas, así como entre los propios estilos que caracterizan y rigen a cada revista.

\section{MATERIAL Y MÉTODO}

Bajo el protocolo de ética en investigación del Comité de Protección de Sujetos Humanos en Investigación Biomédica de París (Le Comité de Protección des Personnes (CPP) de I'lle de France), esta investigación no requiere ser aprobada por un Comité de Ética Científica o el consentimiento de los autores de los artículos originales, debido a su naturaleza puramente descriptiva (Pitak-Arnnop et al., 2009).

Estudio bibliométrico descriptivo observacional de corte transversal cualitativo, aleatorizado simple, en el cual se incluyeron todas las revistas del área odontológica indexadas en la base SciELO (Scientific Electronic Library Online), en idioma español, portugués e inglés, desde Enero a Diciembre del año 2015. Todas las revistas incluidas contaron con acceso online. En el portal web SciELO (www.scielo.org) se realizó la búsqueda a través del enlace "colecciones de revistas", que permitió obtener acceso a los sitios SciELO de cada país, y por lo tanto, a sus colecciones (Argentina, Brasil, Chile, Costa Rica, Cuba, España, México, Perú, Portugal, Bolivia, Paraguay, Uruguay, Sudáfrica y Venezuela). En el sitio SciELO de cada país, se continuó la búsqueda a través del buscador "lista por materia", luego se continuó con el enlace "ciencias de la salud", el que entregó los títulos de las revistas relacionadas con el área odontología, siguiendo la metodología publicada previamente por Cantín \& Aravena (2014). En los casos que no se obtuvo el enlace directo desde el sitio de SciELO, se accedió desde la página oficial de la revista disponible en la base Latindex (www.latindex.org).

La muestra de estudio se constituyó por artículos científicos de todas las revistas, seleccionados a través de un muestreo estratificado por afijación proporcional estimado para cada revista, considerando la publicación total de 1068 artículos el año 2015 (Tabla I). Se incluyeron sólo artículos originales, series o reportes de caso, disponibles para descarga a texto completo. Se excluyeron los estudios en animales y de laboratorio (in vitro o ex vivo), revisiones, opiniones, editoriales, comentarios, erratas, obituarios y cartas al editor. Se seleccionaron todos los artículos que cumplieron los criterios de inclusión dentro de cada revista de manera aleatoria según fascículo o número, hasta que se alcanzó el tamaño de la muestra. Los artículos fueron descargados a texto completo, analizados de manera individual, y las características de citas y referencias fueron consignadas para cada caso.

\section{RESULTADOS}

Fueron revisados 100 artículos pertenecientes a 20 revistas diferentes. El estilo más utilizado para las citas y referencias bibliográficas correspondió al sistema Vancouver (19/20); en el caso de las citas, 
Tabla I. Estimación de tamaño muestral por afijación proporcional para revistas SciELO odontológicas del año 2015.

\begin{tabular}{lcccrrrrc}
\hline Revista & $\mathbf{N i}$ & $\mathbf{p i}$ & $\mathbf{q i}$ & \multicolumn{1}{c}{$\mathbf{N i p i q i}$} & \multicolumn{1}{c}{$\mathbf{p q}$} & $\mathbf{N i} \mathbf{}^{\mathbf{p q}}$ & $\mathbf{n i}$ & $\mathbf{n i}$ \\
\cline { 2 - 8 } S. Afr. Dent. J. & $\mathbf{1 0 6}$ & 0,0996 & 0,9007 & 9,476419 & 0,2989986 & 31,693856 & 10,82329794 & $\mathbf{1 1}$ \\
Acta Odontol. Latinoam. & $\mathbf{2 8}$ & 0,026 & 0,9737 & 0,714836 & 0,1597807 & 4,4738607 & 1,527801726 & $\mathbf{2}$ \\
Braz. Dent. J. & $\mathbf{1 2 0}$ & 0,112 & 0,8876 & 11,968185 & 0,3158082 & 37,896995 & 12,94164008 & $\mathbf{1 3}$ \\
Braz. J. Oral Sci. & $\mathbf{5 3}$ & 0,049 & 0,9503 & 2,499627 & 0,2171699 & 11,51000 & 3,930611362 & $\mathbf{4}$ \\
Braz. Oral Res. & $\mathbf{1 2 4}$ & 0,116 & 0,8838 & 12,725441 & 0,3203506 & 39,723478 & 13,56537502 & $\mathbf{1 4}$ \\
Dental Press J. Orthod. & $\mathbf{9 2}$ & 0,086 & 0,9138 & 7,242407 & 0,2805740 & 25,812816 & 8,814951549 & $\mathbf{9}$ \\
J. Appl. Oral Sci. & $\mathbf{8 8}$ & 0,082 & 0,9176 & 6,653480 & 0,2749686 & 24,197237 & 8,263239221 & $\mathbf{9}$ \\
RGO, Rev. Gaúch. Odontol. & $\mathbf{6 4}$ & 0,059 & 0,9400 & 3,605380 & 0,2373480 & 15,19027 & 5,187404293 & $\mathbf{6}$ \\
Rev. Odontol. UNESP & $\mathbf{6 0}$ & 0,056 & 0,9438 & 3,181416 & 0,2302685 & 13,816113 & 4,718135632 & $\mathbf{5}$ \\
Int. J. Odontostomat. & $\mathbf{7 5}$ & 0,070 & 0,9297 & 4,896990 & 0,2555253 & 19,164401 & 6,544550136 & $\mathbf{7}$ \\
Rev. Clin. Periodoncia Implantol. & $\mathbf{4 7}$ & 0,0440 & 0,9559 & 1,977329 & 0,2051117 & 9,6402524 & 3,292099385 & $\mathbf{4}$ \\
Rehabil. Oral & $\mathbf{8}$ & 0,0074 & 0,9925 & 0,059476 & 0,0862237 & 0,6897896 & 0,235559809 & $\mathbf{1}$ \\
CES odontol. & $\mathbf{1 3}$ & 0,0121 & 0,9878 & 0,156313 & 0,1096545 & 1,4255091 & 0,486804448 & $\mathbf{1}$ \\
Rev. Fac. Odontol. Univ. Antioq. & $\mathbf{5 3}$ & 0,0496 & 0,9503 & 2,499627 & 0,2171699 & 11,51000 & 3,930611362 & $\mathbf{4}$ \\
Rev. Cubana Estomatol. & $\mathbf{3 6}$ & 0,033 & 0,9662 & 1,172579 & 0,1804761 & 6,4971418 & 2,218742383 & $\mathbf{3}$ \\
Av. Odontoestomatol. & $\mathbf{1 0}$ & 0,0093 & 0,9906 & 0,09275 & 0,0963100 & 0,963100 & 0,328894123 & $\mathbf{1}$ \\
Avances en Periodoncia & $\mathbf{2 6}$ & 0,0243 & 0,9756 & 0,617549 & 0,1541165 & 4,0070303 & 1,368381396 & $\mathbf{2}$ \\
Rev. Esp. Cirug. Oraly & $\mathbf{1 8}$ & 0,0168 & 0,9831 & 0,29825 & 0,1287240 & 2,3170326 & 0,7912554 & $\mathbf{1}$ \\
Maxilofac. & $\mathbf{3 1}$ & 0,0290 & 0,9709 & 0,87369 & 0,1678800 & 5,2042801 & 1,777236396 & $\mathbf{2}$ \\
Rev. Odont. Mex. & $\mathbf{1 6}$ & 0,0149 & 0,9850 & 0,23610 & 0,1214777 & 1,9436434 & 0,663744788 & $\mathbf{1}$ \\
Rev. Estomatol. Herediana & & & & & & & & $\mathbf{1 0 0}$ \\
Odontoestomatología & & & & & & & \\
Total & & & & & &
\end{tabular}

todas ellas hicieron uso de números arábicos para citar.

La revista sudafricana, South African Dental Journal (ISSN 0375-1562) realiza las citas colocando números arábicos como superíndice (sin utilizar paréntesis). En el caso de las citas indirectas, cuando el artículo tenía más de dos autores utilizaron la abreviación "et al", en la mayoría de las ocasiones de manera cursivada (en un artículo no se cursivó), la que en ocasiones fue puntuada y en otras no (et al. y et al). En un artículo se observó que cambió esta abreviación por la palabra "colleagues", y en un artículo se observó la cita indirecta sólo incluyendo el autor (es) más el año, pero sin citar con el número superíndice. En el caso de las citas indirectas, al realizar citas de más de dos artículos consecutivos, en algunos casos no utiliza guiones (e.g. 10,11,12 o 5-7,8,9). Por otro lado, en un artículo se detectó que utilizó citas en el "Abstratc" del artículo. Sus referencias bibliográficas siguieron el estilo Vancouver (Tabla II y III).
La revista Argentina, Acta Odontológica Latinoamericana (ISSN 1852-4834) realiza las citas colocando números arábicos como superíndice (sin paréntesis). Cuando realizó citas indirectas de artículos que tenían más de dos autores utilizaron la abreviación "et al", la cual siempre fue puntuada. Esta abreviación se observó en formatos cursivado y no cursivado. Sus referencias bibliográficas siguieron el estilo Vancouver (Tabla II y III).

De las revistas brasileñas, Brazilian Dental Journal (ISSN 1806-4760) realiza las citas colocando números arábicos entre paréntesis (sin superíndice). Cuando realizó citas indirectas de artículos que tenían más de dos autores utilizaron la abreviación "et al.", siempre puntuada, sin cursivar. Brazilian Journal Oral Science (ISSN 1677-3225) realiza las citas colocando números arábicos como superíndice. Cuando realizó citas indirectas de artículos que tenían más de dos autores utilizaron la abreviación "et al.", siempre puntuada, sin cursivar. No aplica norma Vancouver en 
CANTíN, M. \& CHUHUAICURA, P. Estilos de citas y referencias bibliográficas en revistas odontológicas SciELO: Un elemento crítico de la escritura científica.

Int. J. Odontostomat., 10(2):349-357, 2016.

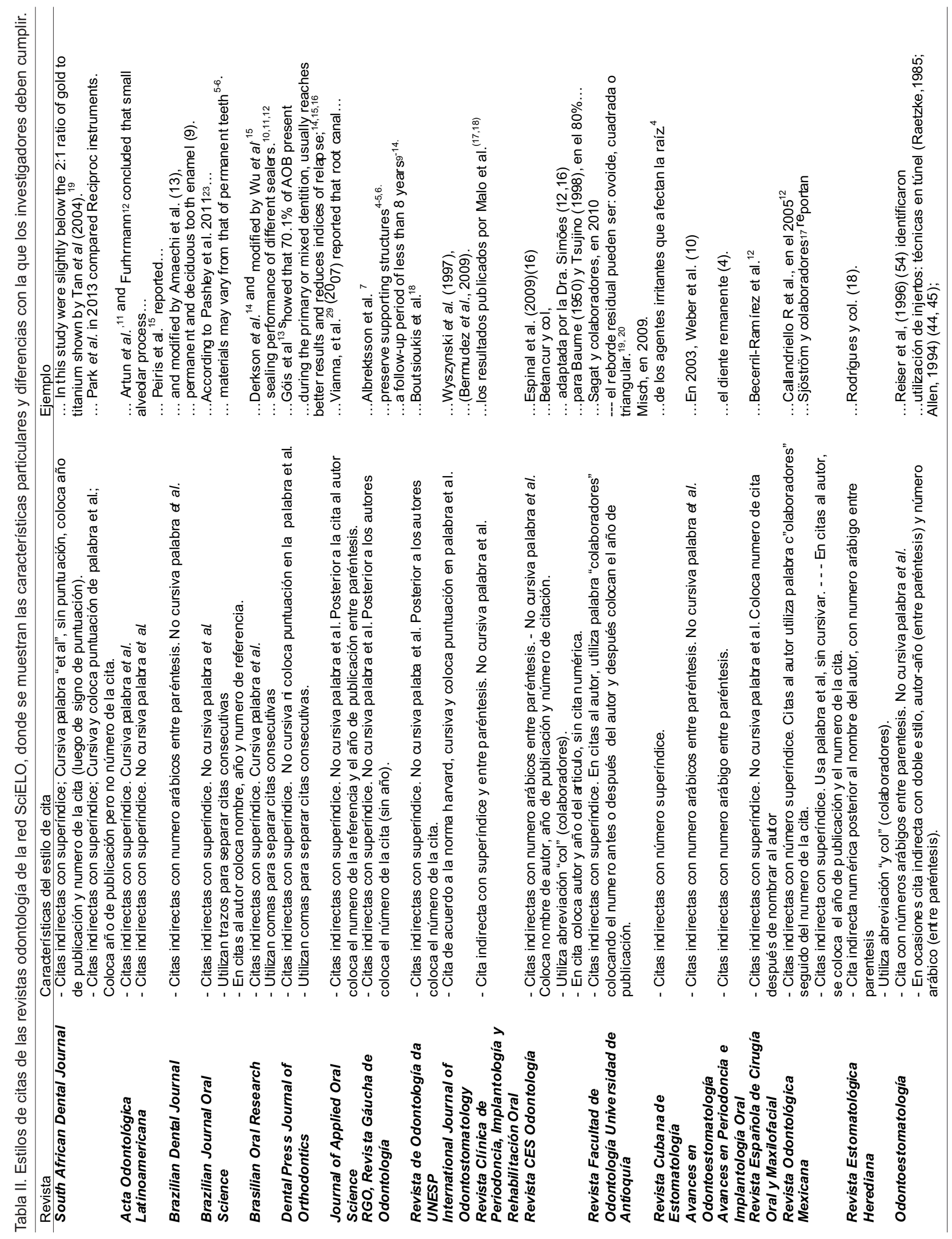


colocar las citas de forma consecutiva (e.g. 5-6). En las citas indirectas coloca nombre (numero de citación y año de publicación, sin utilizar paréntesis en este último). Brasilian Oral Research (ISSN 1807-3107) realiza las citas colocando números arábicos como superíndice, la que se presentó de manera cursivada y no cursivada. Cuando realizó citas indirectas de artículos que tenían más de dos autores utilizaron la abreviación "et al.", la cual siempre fue puntuada. Al realizar citas de más de dos artículos consecutivos, en algunos casos no utiliza guiones (e.g. 10,11,12,13,14 o 19,20,21,22). Dental Press Journal of Orthodonctics (ISSN 2177-6709) realiza las citas colocando números arábicos como superíndice (sin utilizar paréntesis). En el caso de las citas indirectas, cuando el artículo tenía más de dos autores utilizaron la abreviación "et al"; esta abreviación se observó en formatos cursivado y no cursivado, la que en ocasiones fue puntuada y en otras no (et al. y et al). Journal of Applied Oral Science (ISSN 1678-7765) realiza las citas colocando números arábicos como superíndice. No aplica norma Vancouver en colocar citas con numero consecutivos y cronológicos. Cuando realizó citas indirectas de artículos que tenían más de dos autores utilizaron la abreviación "et al.", siempre puntuada, sin cursivar. Posterior a la cita al autor coloca el numero de la referencia y el año de publicación entre paréntesis. RGO, Revista Gáucha de Odontologia (ISSN 1981-8637) realiza las citas colocando números arábicos como superíndice. Cuando realizó citas indirectas de artículos que tenían más de dos autores utilizaron la abreviación "et al.", siempre puntuada, sin cursivar. Revista de Odontología da UNESP (ISSN 1807-2577) realiza las citas colocando números arábicos como superíndice. Cuando realizó citas indirectas de artículos que tenían más de dos autores utilizaron la abreviación "et al.", puntuada, sin cursivar. Al realizar citas de más de dos artículos consecutivos, en algunos casos no utiliza guiones (e.g. 13,14,15,16) (ver Tabla II). Todas siguieron el estilo Vancouver en sus referencias bibliográficas, pero con variaciones (Tabla III).

De las revistas chilenas, International Journal of Odontostomatology (ISSN 0718-381X) cita de acuerdo a la norma Harvard, utilizando el formato Autor(es) y año. Cuando realizó citas de artículos que tenían más de dos autores utilizaron la abreviación "et al.", puntuada y cursivada. Sus referencias también se realizan según esta norma. La Revista Clínica de Periodoncia, Implantología y Rehabilitación Oral (ISSN 0719-0107) realiza la citas colocando números arábicos entre paréntesis y como superíndice. Cuando realizó citas indirectas de artículos que tenían más de dos autores utilizaron la abreviación "et al.", puntuada, sin cursivar. Sus referencias bibliográficas siguieron el estilo Vancouver (Tabla II y III).

De las revistas colombianas, Revista CES Odontología (ISSN 0120-971X) realiza las citas colocando números arábicos entre paréntesis (sin superíndice). Cuando realizó citas indirectas de artículos que tenían más de dos autores utilizaron la abreviación "y col." (y colaboradores), puntuada, sin cursivar. La Revista Facultad de Odontología Universidad de Antioquía (ISSN 0121-246X) realiza las citas colocando números arábicos como superíndice. Cuando realizó citas indirectas de artículos que tenían más de dos autores utilizaron "y colaboradores" (sin abreviar). Sus referencias bibliográficas siguieron el estilo Vancouver (Tabla II y III).

De Cuba, la Revista Cubana de Estomatología (ISSN 1561-297X) realiza la citas colocando números arábicos como superíndice. Sus referencias bibliográficas siguieron el estilo Vancouver (Tabla II y III).

De las revistas españolas, Avances en Odontoestomatología (ISSN 0213-1285) realiza las citas colocando números arábicos entre paréntesis (sin superíndice). Cuando realizó citas indirectas de artículos que tenían más de dos autores utilizaron la abreviación "et al.", siempre puntuada, sin cursivar. Avances en Periodoncia e Implantología Oral (ISSN 16996585) realiza las citas colocando números arábicos entre paréntesis (sin superíndice). Revista Española de Cirugía Oral y Maxilofacial (ISSN 1130-0558) realiza la citas colocando números arábicos como superíndice. En todas, las referencias bibliográficas siguieron el estilo Vancouver (Tabla II y III).

De México, la Revista Odontologica Mexicana (ISSN 1870-199X) realiza las citas colocando números arábicos como superíndice. Cuando realizó citas indirectas de artículos que tenían más de dos autores utilizaron "y colaboradores" (sin abreviar), puntuada, y en otros casos la abreviación "et al.", puntuada, sin cursivar. Sus referencias bibliográficas siguieron el estilo Vancouver (Tabla II y III).

De Perú, la Revista Estomatológica Herediana (ISSN 1019-4355) realiza las citas colocando números arábicos entre paréntesis (sin superíndice). Cuando realizó citas indirectas de artículos que tenían más de dos autores utilizaron la abreviación "y cols." . (y colaboradores), puntuada, sin cursivar. Sus referencias bibliográficas siguieron el estilo Vancouver (Tabla II y III). 
CANTíN, M. \& CHUHUAICURA, P. Estilos de citas y referencias bibliográficas en revistas odontológicas SciELO: Un elemento crítico de la escritura científica. Int. J. Odontostomat., 10(2):349-357, 2016.

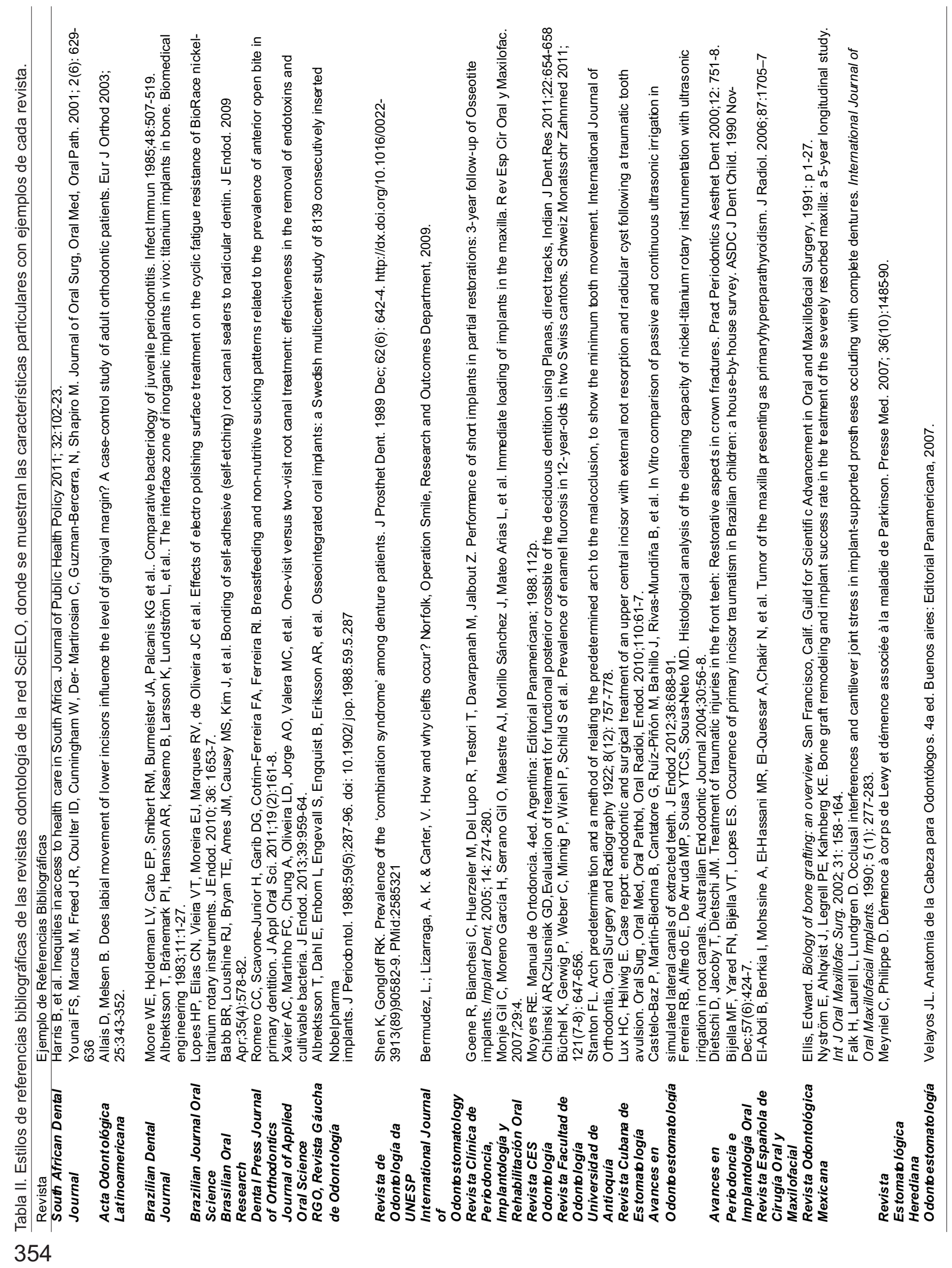


De Uruguay, la revista Odontoestomatología (ISSN 1688-9339) realiza las citas colocando números arábicos entre paréntesis (sin superíndice). Cuando realizó citas indirectas de artículos que tenían más de dos autores utilizaron la abreviación "et al.", puntuada, sin cursivar. Sus referencias bibliográficas siguieron el estilo Vancouver (Tabla II y III).

\section{DISCUSIÓN}

Las revistas odontológicas en su mayoría, al igual que las revistas médicas, siguen los requisitos de uniformidad para manuscritos enviados a revistas biomédicas, estableciendo al sistema Vancouver como el principal estilo para dar formato a las citas y referencias bibliográficas (Bryson, 2012). Sólo una revista, International Journal of Odontostomatology publica sus artículos bajo el sistema Harvard. Así, estos estilos sirven como un sello o de las revistas, pero constituyen una carga de trabajo adicional significativo, ya que la atención a los detalles que se requieren para asegurar que las citas y referencias se ajusten a los estilos individuales de las revistas es fundamental (Harries et al.).

Muchos autores, especialmente los inexpertos, cometen el error de considerar la confección de citas y referencias de un manuscrito una tarea menor. Sin embargo, los equipos editoriales de revistas suelen prestar mucha atención a estos elementos (por razones de reconocimiento científico, derechos de autoría y requisitos de organismos indexadores, sobretodo los que generan métricas), por tanto, si la presentación de citas y referencias no son apropiadas, son inexactas o están en un formato incorrecto, puede ser causa suficiente para devolver (Peh \& Ng) o rechazar un artículo. Ezeala et al. (2013) identificaron y caracterizaron los errores más comunes en los manuscritos originales enviados a revistas médicas, donde el $69 \%$ de los manuscritos tenían defectos en la sección de referencias y su principal error fue la inconsistencias en la citación o no cumplir con el estilo recomendado de la revista (66,7 \%). Mohammad et al. (2008) evaluaron la citación en las cinco principales revistas de cirugía oral y maxilofacial, encontrando un número significativo de errores en cada una de las revistas, demostrando que es necesario un mayor esfuerzo por parte de los autores al dar forma a sus artículos, ya que la preparación de las citas en el formato adecuado es siempre una tarea difícil, sobre todo porque las diferentes publicaciones tienen diferentes estilos.
También es importante considerar que las citas en las diversas revistas analizadas, no sólo se identifican en el cuerpo principal del texto, sino que también pueden aparecer en otras partes del manuscrito como tablas y leyendas de las figuras (Peh \& $\mathrm{Ng}$ ), inclusive en ocasiones en resumen, lo cual podría suceder si es una referencia hacia un artículo fundamental para el objetivo o método del trabajo (que no fueron los casos observados).

En el sistema Harvard, perteneciente a la Universidad de Harvard, las citas (indirectas) se realizan colocando el apellido del autor y el año de publicación entre paréntesis, por ejemplo: "(Kuo, 2016)". Como alternativa, el nombre del autor puede ser parte de la frase (cita directa o indirecta), en cuyo caso, sólo el año irá entre paréntesis, por ejemplo: "Kuo (2016) demostró que ...". Si hay dos autores, se dan dos nombres, por ejemplo: (Kuo y Malbec, 2016). Si hay más de dos autores, el nombre del primer autor es citado, seguido de "et al.", por ejemplo (Kuo et al., 2016). Si se citan varias referencias en conjunto en el texto, se ordenarán cronológicamente y separadas por punto y coma, por ejemplo (Albert, 2012; Kuo y Malbec, 2015, Kuo et al., 2016). Al final del documento, las referencias bibliográficas se ordenarán alfabéticamente.

El sistema de Vancouver fue creado en 1978 por un grupo de editores de revistas biomédicas que se reunió en la ciudad de Vancouver y decidió prescribir proposiciones técnicas uniformes para su publicación. Fue adoptado en el 1979 por la Biblioteca Nacional de Medicina en Bethesda y luego por el Comité Internacional de Editores de Revistas Médicas (ICMJE, 2016), cuya revisión en 1982, entró en la solicitud oficial de 300 revistas biomédicas internacionales, incluyendo a revistas odontológicas (Masic). En este sistema, las referencias son numeradas consecutivamente en el orden en que se citan en el texto y se identifican por números arábicos, apareciendo en el texto, por lo general, ya sea entre paréntesis, por ejemplo: $(1,2)$, o como superíndices, por ejemplo: ${ }^{1,2}$, o incluso de manera conjunta como superíndices entre paréntesis ${ }^{(1,2)}$ (Peh \& Ng). Esto confirma lo observado en las revistas odontológicas que siguen el sistema Vancouver, el cuál varió entre las revistas de diferentes países, entre revistas de un mismo país e incluso dentro de cada revista en algunas ocasiones. Sin embargo, el observar diferencias de estilos dentro de una misma revista o artículo, nos muestra más una inconsistencia que una variación de estilo. 
Así, las revistas odontológicas se comportan igual que otras biomédicas, donde las pautas o instrucciones de cada revista especifican uno de estos dos sistemas, pero añaden pequeñas variaciones, haciendo que cada estilo de la revista sea único; en efecto, puede haber tantos estilos de referencia, como revistas (Harries et al.). Estas variaciones menores en el diseño / estilo con respecto a la puntuación, uso de fuentes (por ejemplo, cursiva, negrita), superíndices y tipo de paréntesis (McD Taylor, 2002), el espaciamiento y abreviaturas, etc., que por lo general se pueden encontrar en las "Instrucciones a los Autores" o "Directrices de Autor" de cada revista, muchas inclusive con ejemplos ilustrativos (Peh \& Ng). Al preparar un manuscrito para su publicación, los autores deben cumplir con el formato de referencia de la revista a la que deseen enviar el artículo. Muchas de las revistas odontológicas SciELO tienen ejemplos de referencia en su apartado de introducción a los autores, o bien, puede tomarse como ejemplo las citas y referencias utilizadas en cualquier artículo publicado por esa revista (idealmente del número/fascículo más actual).

En la actualidad, la difícil tarea de citar y referenciar puede ser apoyada por gestores de refe- rencia como RefWorks o Endnote que realizan enlaces con el procesador de textos, Papers, que realiza el enlace con internet y las referencias DOI para encontrar todos los detalles del artículo, o Zotero y Mendeley, que funcionan como herramientas para navegadores web (estos últimos inclusive libres y gratuitos) (Bryson). Una desventaja de todos los gestores, es que no son fáciles de adaptar a las diferentes revistas, y todavía se requieren cambios manuales para garantizar la conformidad con las normas de cada revista (Harries et al.). Los equipos editoriales igual revisan y corrigen las citas y referencias elaboradas por gestores, con el fin de garantizar la conformidad con el estilo o sus variaciones requerido por cada revista (Friedberg, 2005).

Cualquiera sea el sistema que se elija, es importante utilizarlo de una manera consistente (Dwyer, 1995) y se debe ajustar estrictamente al estilo de la revista, el que debe figurar en la Guía para los autores (Vetto, 2014; Lefor \& Maeno, 2016). La responsabilidad final de la exactitud en las citas y referencias bibliográficas es de los autores, por lo que deben preocuparse de ello al redactar el artículo, en su revisión final, así como en las pruebas de imprenta.

CANTíN, M. \& CHUHUAICURA, P. Styles for citations and referencing in dental SciELO journals: A critical element of scientific writing. Int. J. Odontostomat., 10(2):349-357, 2016.

ABSTRACT: One of the most common problems in scientific journals, it is the failure of the styles to make citations and references due to carelessness or ignorance of the different systems and their characteristics. The aim was to review the different styles of citations and references used by dental journals indexed in SciELO, to describe the most used and highlight the differences between journals. One hundred articles randomly selected by proportional allocation, belonging to 20 different journals were reviewed. The most commonly used format for citations and references corresponded to the Vancouver system. Only a journal, International Journal of Odontostomatology publishes his articles under the Harvard system. These styles serve as a hallmark for journals, but they are a significant additional workload as the attention to detail required to ensure that citations and references fit individual styles of the journals is fundamental. Whatever the style of the journals, it is important to use it in a consistent manner. The responsibility for the accuracy in citations and references belongs to the authors, so they should worry about it in drafting version, its final revision, as well as proofs of the article.

KEY WORDS: references, styles of references, citations, Vancouver, Harvard, scientific writing.

\section{REFERENCIAS BIBLIOGRÁFICAS}

Bryson, D. Using research papers: citations, referencing and plagiarism. J. Vis. Commun. Med., 35(2):82-4, 2012.

Cantín, M. \& Aravena, Y. Las revistas odontológicas en la base SciELO: una mirada bibliométrica. Int. J. Odontostomat., 8(2):215-20, 2014.

Dwyer, M. A guide to the Harvard referencing system. Br. J. Nurs., 4(10):599-602, 1995.
Ezeala, C.; Nweke, I. \& Ezeala, M. Common errors in manuscripts submitted to medical science journals. Ann. Med. Health Sci. Res., 3(3):376-9, 2013.

Friedberg, E. C. Call for a cull of pointlessly different reference styles. Nature, 437(7063):1232, 2005.

Harries, A. D.; Kumar, A. M.; Satyanarayana, S.; Bissell, K.; Hinderaker, S. G.; Edginton, M.; Reid, A. J. \& Zachariah, 
CANTíN, M. \& CHUHUAICURA, P. Estilos de citas y referencias bibliográficas en revistas odontológicas SciELO: Un elemento crítico de la escritura científica. Int. J. Odontostomat., 10(2):349-357, 2016

R. References for scientific papers: why not standardise to one global style? Public Health Action, 3(3):255-7, 2013.

International Committee of Medical Journal Editors. Uniform requirements for manuscripts submitted to biomedical journals: writing and editing for publication. 2016. Disponible en: http://www.icmje.org

Kali, A. Reference management: Acritical element of scientific writing. J. Adv. Pharm. Technol. Res., 7(1):27-9, 2016.

Laskin, D. M. Paying attention to details. J. Oral Maxillofac. Surg., 54(2):133, 1996.

Lefor, A. K. \& Maeno, M. Preparing scientific papers, posters, and slides. J. Surg. Educ., 73(2):286-90, 2016.

Masic, I. The importance of proper citation of references in biomedical articles. Acta Inform. Med., 21(3):148-55, 2013.

McD Taylor, D. The appropriate use of references in a scientific research paper. Emerg. Med. (Fremantle), 14(2):166-70, 2002.

Mohammad, A. E. \& Laskin, D. M. Citation accuracy in the oral and maxillofacial surgery literature. J. Oral Maxillofac. Surg., 66(1):3-6, 2008.

Peh, W. C. \& Ng, K. H. Preparing the references. Singapore Med. J., 50(7):659-61; quiz 662, 2009.

Pitak-Arnnop, P.; Sader, R.; Hervé, C.; Dhanuthai, K.; Bertrand, J. C. \& Hemprich, A. Reporting of ethical protection in recent oral and maxillofacial surgery research involving human subjects. Int. J. Oral Maxillofac. Surg., 38(7):707-12, 2009.

Reyes, $\mathrm{H}$. The references in articles published in biomedical journals. Rev. Med. Chil., 129(4):343-5, 2001.

Riordan, L. Modern-day considerations for references in scientific writing. J. Am. Osteopath. Assoc., 112(8):5679, 2012.

Vetto, J. T. Short and sweet: a short course on concise medical writing. J. Cancer Educ., 29(1):194-5, 2014.
Dirección para Correspondencia:

Mario Cantín L.

Facultad de Odontología

Universidad de la Frontera

Manuel Montt 112

Casilla 54-D

Temuco

CHILE

Email: mario.cantin@ufrontera.cl

Recibido : 16-05-2016

Aceptado: 27-07-2016 